\title{
Correction to: Maternal blood glucose level and offspring glucose-insulin homeostasis: what is the role of offspring adiposity?
}

\author{
Ellen C. Francis ${ }^{1,2}$ (D) $\cdot$ Dana Dabelea ${ }^{1,2,3}$ (D) $\cdot$ Brandy M. Ringham ${ }^{1} \cdot$ Katherine A. Sauder $^{1,2,3}$ (D) Wei Perng $^{1,2}$ (D)
}

Published online: 23 March 2021

(C) Springer-Verlag GmbH Germany, part of Springer Nature 2021

\section{Correction to: Diabetologia}

https://doi.org/10.1007/s00125-020-05294-2

Unfortunately, $\mathrm{HbA}_{1 \mathrm{c}}$ values were incorrectly converted from per cent to $\mathrm{mmol} / \mathrm{mol}$ in this paper. The corrected text is provided below, along with sections of Table 1, showing corrected $\mathrm{HbA}_{1 \mathrm{c}}$ data, and Tables 2 and 3, showing corrected estimates of association per $1 \mathrm{mmol} / \mathrm{mol} \mathrm{HbA}_{1 \mathrm{c}}$.

Following the amendments to the $\mathrm{HbA}_{1 \mathrm{c}}$ values in $\mathrm{mmol} / \mathrm{mol}$, the authors would like to note that the magnitude and direction of effects, as well as the $p$ values reported, have not changed and that the overall findings of the study remain unaltered.

\section{Results}

\section{Characteristics}

The mean \pm SD age of the women at enrolment was $28.7 \pm$ 6.0 years, most were non-Hispanic white $(58.6 \%)$ and the median (range) of maternal $\mathrm{HbA}_{1 \mathrm{c}}$ was $31.2 \mathrm{mmol} / \mathrm{mol}$ (16.9-45.4) (5.0\% [3.7-6.3]).

\section{Assessment of offspring adiposity as mediator}

This is likely due to the fact that, despite having a relatively normal distribution and meeting assumptions of multivariate normality, several women in our sample had a similar $\mathrm{HbA}_{1 \mathrm{c}}$ $\left(n=98\right.$ [28.4\%] with $\mathrm{HbA}_{1 \mathrm{c}}=32.2 \mathrm{mmol} / \mathrm{mol}[5.1 \%]$ or $31.2 \mathrm{mmol} / \mathrm{mol}[5.0 \%])$, thereby reducing variability in the explanatory variable.

Publisher's note Springer Nature remains neutral with regard to jurisdictional claims in published maps and institutional affiliations.

2 Department of Epidemiology, Colorado School of Public Health, University of Colorado Denver Anschutz Medical Campus, Aurora, CO, USA

Ellen.Francis@CUAnschutz.edu

Lifecourse Epidemiology of Adiposity and Diabetes (LEAD) Center, University of Colorado Denver Anschutz Medical Campus,

Aurora, CO, USA

\footnotetext{
Department of Pediatrics, School of Medicine, University of Colorado Denver Anschutz Medical Campus, Aurora, CO, USA
} 
Table 1 Bivariate associations of maternal and offspring characteristics with maternal $\mathrm{HbA}_{1 \mathrm{c}}$

\begin{tabular}{lcccc}
\hline Characteristic & Overall $(n=345)$ & $\mathrm{HbA}$ & & $p$ value \\
\cline { 3 - 5 } & & Tertile 1 & Tertile 2 & Tertile 3 \\
\hline Maternal characteristics & & & & \\
$\mathrm{HbA}_{1 \mathrm{c}}$, mmol/mol, median (range) & $31.2(16.9-45.4)$ & $29.0(17.0-30.1)$ & $31.7(31.2-32.2)$ & $34.4(33.3-45.5)$ \\
$\mathrm{HbA}_{1 \mathrm{c}}, \%$, median (range) & $5.0(3.7-6.3)$ & $4.8(3.7-4.9)$ & $5.1(5.0-5.1)$ & $5.3(5.2-6.3)$ \\
\hline
\end{tabular}

Table 2 Total effect of maternal $\mathrm{HbA}_{1 \mathrm{c}}$ on offspring biomarkers of glucose-insulin homeostasis at age 4-7 years

\begin{tabular}{|c|c|c|c|c|}
\hline \multirow[t]{2}{*}{ Biomarker } & \multicolumn{2}{|l|}{ Model 1} & \multicolumn{2}{|l|}{ Model 2} \\
\hline & $\beta(95 \% \mathrm{CI})$ & $p$ value & $\beta(95 \% \mathrm{CI})$ & $p$ value \\
\hline \multicolumn{5}{|l|}{ Glucose, mmol/1 } \\
\hline $\mathrm{HbA}_{1 \mathrm{c}} \mathrm{T} 2$ vs $\mathrm{T} 1$ & $0.04(-0.05,0.13)$ & & $0.04(-0.05,0.13)$ & \\
\hline $\mathrm{HbA}_{1 \mathrm{c}} \mathrm{T} 3$ vs $\mathrm{T} 1$ & $0.17(0.08,0.26)^{*}$ & $<0.001^{\mathrm{a} *}$ & $0.16(0.06,0.25)^{*}$ & $<0.001^{\mathrm{a} *}$ \\
\hline $\mathrm{HbA}_{1 \mathrm{c}}($ per $1 \mathrm{mmol} / \mathrm{mol})$ & $0.009(-0.003,0.020)$ & $0.14^{\mathrm{b}}$ & $0.007(-0.004,0.018)$ & $0.23^{\mathrm{b}}$ \\
\hline \multicolumn{5}{|l|}{$1 /($ fasting insulin), $\mathrm{pmol} / 1$} \\
\hline $\mathrm{HbA}_{1 \mathrm{c}} \mathrm{T} 2$ vs $\mathrm{T} 1$ & $-0.001(-0.004,0.003)$ & & $0.000(-0.004,0.003)$ & \\
\hline $\mathrm{HbA}_{1 \mathrm{c}} \mathrm{T} 3$ vs $\mathrm{T} 1$ & $-0.004(-0.008,-0.000)^{*}$ & $0.04^{\mathrm{a} *}$ & $-0.004(-0.008,0.000)$ & $0.06^{\mathrm{a}}$ \\
\hline $\mathrm{HbA}_{1 \mathrm{c}}($ per $1 \mathrm{mmol} / \mathrm{mol})$ & $0.000(-0.001,-0.000)^{*}$ & $0.04^{\mathrm{b} *}$ & $0.000(-0.001,0.000)$ & $0.07^{\mathrm{b}}$ \\
\hline \multicolumn{5}{|l|}{ HOMA2-IR } \\
\hline $\mathrm{HbA}_{1 \mathrm{c}} \mathrm{T} 2$ vs $\mathrm{T} 1$ & $0.05(-0.05,0.15)$ & & $0.04(-0.06,0.14)$ & \\
\hline $\mathrm{HbA}_{1 \mathrm{c}} \mathrm{T} 3$ vs $\mathrm{T} 1$ & $0.09(-0.00,0.20)$ & $0.08^{\mathrm{a}}$ & $0.08(-0.02,0.19)$ & $0.11^{\mathrm{a}}$ \\
\hline $\mathrm{HbA}_{1 \mathrm{c}}($ per $1 \mathrm{mmol} / \mathrm{mol})$ & $0.008(-0.005,0.020)$ & $0.21^{\mathrm{b}}$ & $0.006(-0.002,0.019)$ & $0.32^{\mathrm{b}}$ \\
\hline \multicolumn{5}{|l|}{ HOMA2-B } \\
\hline $\mathrm{HbA}_{1 \mathrm{c}} \mathrm{T} 2$ vs $\mathrm{T} 1$ & $1.10(-5.65,7.85)$ & & $0.80(-5.97,7.58)$ & \\
\hline $\mathrm{HbA}_{1 \mathrm{c}} \mathrm{T} 3$ vs $\mathrm{T} 1$ & $0.99(-6.11,8.08)$ & $0.78^{\mathrm{a}}$ & $0.62(-6.52,7.76)$ & $0.86^{\mathrm{a}}$ \\
\hline $\mathrm{HbA}_{1 \mathrm{c}}($ per $1 \mathrm{mmol} / \mathrm{mol})$ & $0.460(-0.388,1.308)$ & $0.29^{\mathrm{b}}$ & $0.411(-0.447,1.269)$ & $0.35^{\mathrm{b}}$ \\
\hline
\end{tabular}

Model 1 adjusted for maternal race/ethnicity, child's sex and age at assessment; Model 2 included the same adjustments as Model 1, and was additionally adjusted for pre-pregnancy BMI

${ }^{\mathrm{a}} p$ trend

${ }^{\mathrm{b}} p$ difference

* Statistical significance at $\alpha=0.05$

$\mathrm{T} 1$, tertile 1 ; T2, tertile 2 ; T3, tertile 3 


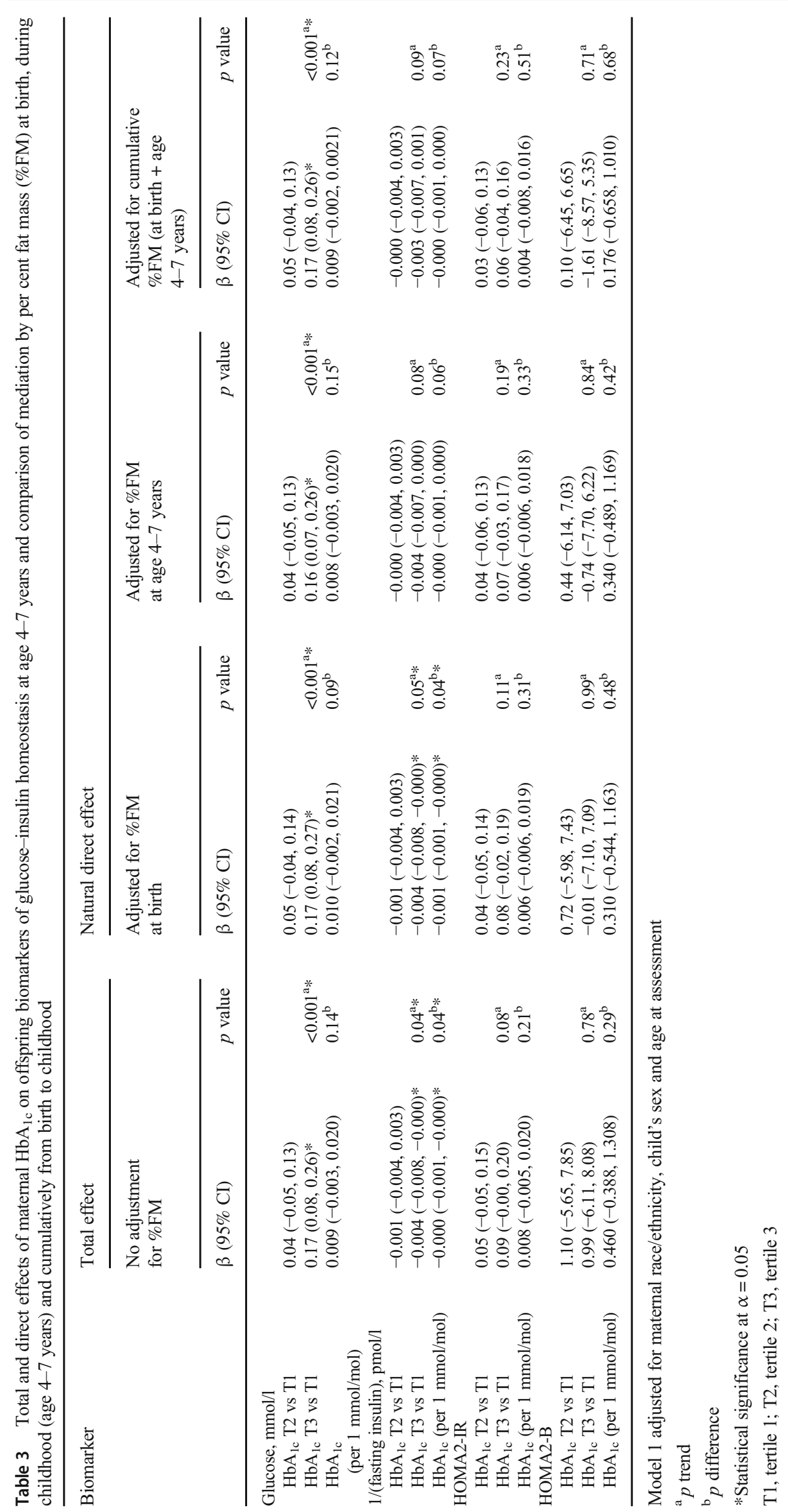

